\title{
Cluster Preface: Non-Covalent Interactions in Asymmetric Catalysis
}

\author{
Robert J. Phipps*
}

Department of Chemistry, University of Cambridge, Lensfield

Road, Cambridge, CB2 1EW, United Kingdom.

rip71@cam.ac.uk

\section{Received:
Accepted:
Published online:}

Abstract The successful harnessing of non-covalent interactions to activate functional groups and control selectivity in chemical reactions is a relatively recent phenomenon in synthetic chemistry, but one that has delivered ground-breaking results, particularly over the last decade, and still bears enormous promise. This Cluster brings together seven manuscripts which represent cutting edge advances in both understanding and applications of non-covalent interactions for asymmetric catalysis.

Key words Non-covalent interactions, organocatalysis, hydrogen-bonding catalysis, supramolecular catalysis, asymmetric catalysis

Catalysis is arguably the most powerful tool in synthetic chemistry, enabling myriad new reactivity, controlling multiple aspects of selectivity and allowing newly efficient processes to be developed. Focusing on the field of asymmetric catalysis, the demand for single enantiomers of chiral compounds for a range of applications from pharmaceuticals to materials science continues unabated. Over the last 50 years we have come a long way from the earliest approaches, which often commenced from the chiral pool. Chiral auxiliary approaches provided powerful new approaches to single enantiomers, but required use of stoichiometric chiral material and extra synthetic manipulations. It was subsequent breakthroughs in asymmetric hydrogenation, asymmetric oxidation and asymmetric Lewis acid catalysis which cemented catalysis as the leading approach to single enantiomer compounds. ${ }^{1}$ These approaches most commonly rely on a metal center bound to a chiral ligand through dative covalent bonds, by now a well-established and successful strategy. Whilst non-covalent interactions had been suggested to aid stereocontrol in some important processes, such as Noyori transfer hydrogenation (substrate-ligand hydrogen bond $)^{2}$ and oxazaborolidine-catalyzed reactions (formyl hydrogen bond) ${ }^{3}$, they were generally regarded as secondary controlling elements. This is somewhat in contrast to Nature's methods for forming stereocenters through enzyme catalysis in which it is more often non-covalent interactions that are driving the mechanism of catalysis at the enzyme active site, using a network of interactions to preassemble reactants and stabilize transition states. ${ }^{4}$ A variety of non-covalent interactions have been shown to be crucial, including electrostatic interactions, hydrogen bonds, hydrophobic effects, $\pi$-cation and $\pi$ - $\pi$ interactions. Whilst important early work employing hydrogen bonding catalysts was done in the late $1990 \mathrm{~s},{ }^{5}$ the divide between Nature's approach and our own was narrowed with the rapid development since the early 2000s of the field of organocatalysis. ${ }^{6}$ Using this approach, small organic molecules have been demonstrated to be powerful catalysts for asymmetric transformations, demonstrating that the more classical metal-chiral ligand combination is not the only viable approach. ${ }^{7}$ Importantly, selectivity in the prototypical organocatalytic transformation, the prolinecatalyzed aldol reaction, was found to be heavily dependent on a key hydrogen bonding interaction between the carboxylic acid and the acceptor aldehyde, clearly demonstrating the potential of non-covalent interactions. ${ }^{8}$ A distinct branch of organocatalysis quickly emerged in which hydrogen bonds are the sole activating and controlling interactions in the mode of catalysis. ${ }^{9}$ This has been exploited in numerous studies with Brønsted acids, ${ }^{10}$ such as chiral phosphoric acids, ${ }^{11}$ as well as dual hydrogen-bond donors exemplified by thioureas. ${ }^{12}$ Using this activation mode, a vast number of highly enantioselective reactions have been developed over the last decade, with bifunctional hydrogen-bonding catalysis also playing an important role. Tellingly, this approach now starts to look more akin to enzyme catalysis, with a multifunctional chiral organic molecule assembling the reactants and controlling the transition state using primarily non-covalent interactions. Indeed, highly efficient asymmetric catalysis based on another key noncovalent interaction, ion pairing, has been known since the mid1980s in the form of asymmetric phase transfer catalysis using chiral cations to ion pair with reactive anionic intermediates. ${ }^{13}$ More recently there have been a number of advances in the charge inverted scenario, where chiral anions ion pair with cationic reactive intermediates, metal catalysts or reagents. ${ }^{14} \mathrm{~A}$ 
twist on the classical metal-chiral ligand approach involves incorporating a ligand assembled by ion pairing, potentially allowing rapid ligand screening. ${ }^{15}$ Progress has also been made in exploring the use of less common non-covalent interactions to asymmetric catalysis and in this arena there remain many exciting possibilities. For example, $\pi$-cation interactions have been shown to be highly effective in achieving stereocontrol in polycyclization reactions, ${ }^{16}$ as well as in other applications. ${ }^{17}$ Recent work has shown that the more elusive $\pi$-anion interaction has great potential as a new tool for asymmetric catalysis. ${ }^{18}$ For some time, supramolecular chemists have designed 'guests' with well defined, hydrophobic cavities which seek to emulate the active site of enzymes in order to bind hosts through the hydrophobic effect. ${ }^{19}$ Encouraging progress has been made into adapting this approach to encompass catalytic asymmetric reactions using chiral hosts. ${ }^{20}$ Indeed, the application of non-covalent interactions to control various aspects of selectivity in chemical reactions is sometimes referred to as 'supramolecular catalysis' and a recent comprehensive review covers this broad remit, providing opportunity for inspiration. ${ }^{21}$

The aim of this Cluster is to bring together manuscripts from leaders in this field, which represent cutting edge advances in both understanding and applications of non-covalent interactions for asymmetric catalysis.

Professor Benjamin List and co-workers present an Account of their recent work on the activation of ketones and carboxylic acids using binol phosphates by way of 'asymmetric enol catalysis'. Professor Kazuaki Ishihara and co-workers report a supramolecular Lewis acid catalyst architecture assembled through CN-B and PO-B coordination bonds, in which a $\mathrm{CH}-\mathrm{O}$ hydrogen bond is hypothesized to be crucial in order to achieve high enantioselectivity in the targeted Diels-Alder reactions. Professors Ryan Gilmour and Ken Houk and co-workers demonstrate that tandem stabilizing electrostatic and hyperconjugative interactions can be powerful controllers of conformation and thus enantioselectivity, with extended conformational control through a carbon framework. A contribution from Professor Stefan Matile's group offers further insight into their recent investigations into scaffolds for anion- $\pi$ catalysis by systematic expansion of the $\pi$-acidic surface. Professor Yong Huang and co-workers report on the use of $\mathrm{N}$ Heterocyclic Carbenes as non-covalent enantioselective organocatalysts, a novel approach that sits in sharp contrast to NHCs more established role as covalent organocatalysts. A report from Professor Thorsten Bach's group demonstrates the potential of using multiple non-covalent interactions as the basis for a bifunctional ligand strategy for control of enantioselectivity in photochemical reactions, in which a transition metal is also involved in the catalytic system. Professor Takashi Ooi and co-workers present insightful work which probes the stoichiometry of their ion-pairing chiral ligands for palladium, which shows that the stoichiometry of the chiral anion can have significant effect on enantioinduction.

We hope that the manuscripts presented in this Cluster demonstrate some of the diverse and exciting ways in which non-covalent interactions can be used in catalytic enantioselective systems, and thus highlight the remarkable potential that remains in this ever expanding field.

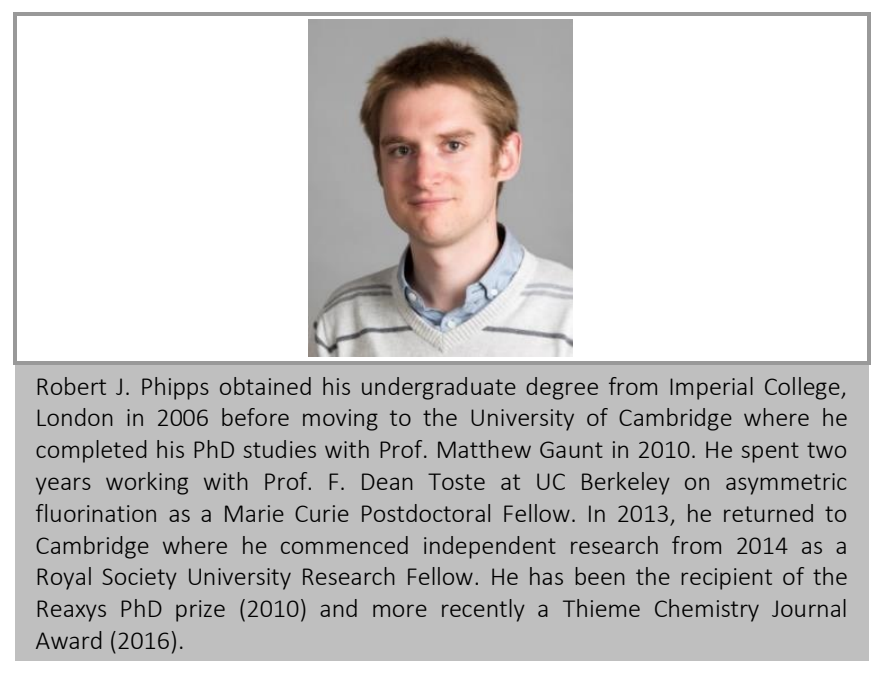

\section{Acknowledgment}

RJP is grateful to the Royal Society for a University Research Fellowship and to Professor Steven V. Ley for advice and useful discussions.

\section{References and Notes}

(1) In Catalytic Asymmetric Synthesis; Third ed.; Ojima, I., Ed.; Wiley: Weinheim, 2010.

(2) Noyori, R. Angew. Chem. Int. Ed. 2002, 41, 2008.

(3) Corey, E. J.; Lee, T. W. Chem. Commun. 2001, 1321

(4) Knowles, R. R.; Jacobsen, E. N. Proc. Natl. Acad. Sci. USA 2010, 107, 20678.

(5) Corey, E. J.; Grogan, M. J. Org. Lett. 1999, 1, 157; Miller, S. J.; Copeland, G. T.; Papaioannou, N.; Horstmann, T. E.; Ruel, E. M. J. Am. Chem. Soc. 1998, 120, 1629;Sigman, M. S.; Jacobsen, E. N. J. Am. Chem. Soc. 1998, 120, 4901.

(6) Dalko, P. I. Comprehensive Enantioselective Organocatalysis; Wiley, 2013.

(7) MacMillan, D. W. C. Nature 2008, 455, 304.

(8) Bahmanyar, S.; Houk, K. N.; Martin, H. J.; List, B. J. Am. Chem. Soc. 2003, 125, 2475.

(9) Taylor, M. S.; Jacobsen, E. N. Angew. Chem. Int. Ed. 2006, 45, 1520.

(10) Turkmen, Y., E.; Zhu, Y.; Rawal, V. H. In Comprehensive Enantioselective Organocatalysis; Dalko, P. I., Ed.; Wiley: Weiheim, 2013; Vol. 1.

(11) Parmar, D.; Sugiono, E.; Raja, S.; Rueping, M. Chem. Rev. 2014, 114, 9047.

(12) Doyle, A. G.; Jacobsen, E. N. Chem. Rev. 2007, 107, 5713; Schreiner, P. R.; Jakab, G. In Comprehensive Enantioselective Organocatalysis; Dalko, P. I., Ed.; Wiley: Weiheim, 2013; Vol. 1.

(13) Dolling, U. H.; Davis, P.; Grabowski, E. J. J. J. Am. Chem. Soc. 1984, 106, 446; Ooi, T.; Maruoka, K. Angew. Chem. Int. Ed. 2007, 46, 4222; Shirakawa, S.; Maruoka, K. Angew. Chem. Int. Ed. 2013, 52, 4312.

(14) Phipps, R. J.; Hamilton, G. L.; Toste, F. D. Nature Chem. 2012, 4, 603; Mahlau, M.; List, B. Angew. Chem. Int. Ed. 2013, 52, 518; Brak, K.; Jacobsen, E. N. Angew. Chem. Int. Ed. 2013, 52, 534.

(15) Ohmatsu, K.; Ito, M.; Kunieda, T.; Ooi, T. Nature Chem 2012, 4, 473; Ohmatsu, K.; Hara, Y.; Ooi, T. Chemical Science 2014, 5, 3645.

(16) Knowles, R. R.; Lin, S.; Jacobsen, E. N. J. Am. Chem. Soc. 2010, 132, 5030.

(17) Zhang Q; Tiefenbacher K Nature Chem. 2015, 7, 197; Yamada, S.; Fossey, J. S. Org. Biomol. Chem. 2011, 9, 7275.

(18) Zhao, Y.; Cotelle, Y.; Avestro, A.-J.; Sakai, N.; Matile, S. J. Am. Chem. Soc. 2015, 137, 11582.

(19) Raynal, M.; Ballester, P.; Vidal-Ferran, A.; van Leeuwen, P. W. N. M. Chem. Soc. Rev. 2014, 43, 1734.

(20) Zhao, C.; Sun, Q.-F.; Hart-Cooper, W. M.; DiPasquale, A. G.; Toste, F. D.; Bergman, R. G.; Raymond, K. N. J. Am. Chem. Soc. 2013, 135, 18802. 
(21) Raynal, M.; Ballester, P.; Vidal-Ferran, A.; van Leeuwen, P. W. N. M. Chem. Soc. Rev. 2014, 43, 1660.

\section{Checklist (have these on hand for manuscript submission in ScholarOne):}

- cover letter, including a statement of the work's significance

- full mailing address, telephone and fax numbers, and e-mail address of the corresponding author

- email address for each author

- original Word file

- original graphics files zipped into one zip file

- eye-catching graphical abstract as an individual file

- 5-8 key words

- separate Supporting Information file

- separate zipped Primary Data files including cover sheet (optional)

\section{Useful links:}

- SYNLETT homepage

- SYNLETT information and tools for authors

- Graphical abstract samples (PDF file download)

- What is "Primary Data"?

- ScholarOne (manuscript submission) 